\title{
NEURAL CONTROL OF VENTILATION PREVENTS BOTH OVER-DISTENSION AND DE- RECRUITMENT OF EXPERIMENTALLY INJURED LUNGS
}

Lukas Brander ${ }^{1,7}$, Onnen Moerer ${ }^{1,5}$, Göran Hedenstierna ${ }^{2}$, Jennifer Beck ${ }^{3,4,8}$, Jukka Takala ${ }^{6}$, Arthur S Slutsky ${ }^{1,4}$, Christer Sinderby ${ }^{1,4,8}$

${ }^{1}$ Interdepartmental Division of Critical Care Medicine, University of Toronto, Department of Critical Care Medicine, St. Michael's Hospital, Toronto, Canada

${ }^{2}$ Department of Medical Sciences, Clinical Physiology, University of Uppsala, Uppsala, Sweden.

${ }^{3}$ Department of Pediatrics, University of Toronto, Toronto, Canada

${ }^{4}$ Keenan Research Centre for Biomedical Science and Li Ka Shing Knowledge Institute of St. Michael's Hospital, Toronto, Canada

5 Department of Anaesthesiology, Emergency and Critical Care Medicine, University of Göttingen, Germany

${ }^{6}$ Department of Intensive Care Medicine, Bern University Hospital - Inselspital, and University of Bern, Switzerland

${ }^{7}$ Department of Intensive Care Medicine, Cantonal Hospital of Lucerne, Switzerland

${ }^{8}$ Member, Institute for Biomedical Engineering and Science Technology (iBEST) at Ryerson University and St-Michael's Hospital 


\title{
Correspondence and requests for reprints should be addressed to
}

\author{
Lukas Brander, MD \\ Department of Intensive Care Medicine \\ Cantonal Hospital of Lucerne \\ 6000 Lucerne, 16 \\ Switzerland \\ e-mail: lukas.brander@luks.ch
}

The work has been performed at the Department of Clinical Physiology, University of Uppsala, Sweden; and at St. Michaels's Hospital, University of Toronto; Canada

Grant support: Supported by St. Michael's Hospital, Toronto, Canada; the Canada Foundation for Innovation (CFI); and the R. Samuel McLaughlin Foundation. Also supported by grants from the Swedish Research Council nr 5315. Lukas Brander held postdoctoral fellowships of the Swiss Foundation for Grants in Biology and Medicine (SFGBM) provided by Novartis AG in collaboration with the Swiss National Science Foundation (SNSF) and of the Division of Respirology at the University of Toronto provided by Merck-Frosst. 


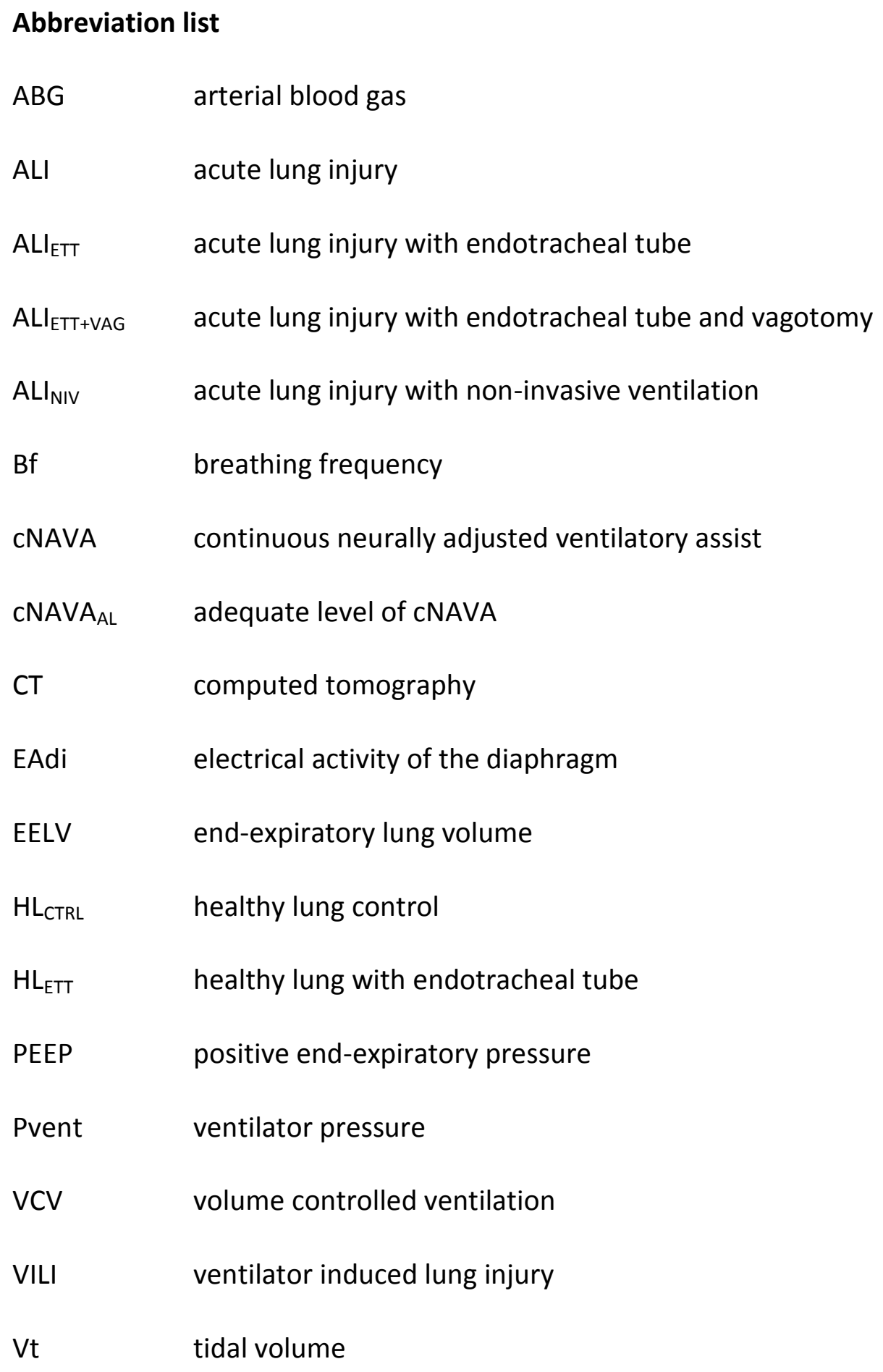


Running title: Continuous neurally adjusted ventilatory assist (cNAVA)

Keywords: acute respiratory failure, mechanical ventilation, diaphragm electrical activity, neural control of breathing, CT scan

Part of the results have been presented in abstract format at the annual meetings of the American Thoracic Society (ATS) in San Francisco, USA, May 18-23, 2007 and of the Swiss Society of Intensive Care Medicine (SGI) in Interlaken, Switzerland, November 1-3, 2007.

Total word count of the manuscript: 2940

Acknowledgment: We are indebted to Norman Comtois, for his excellent technical assistance and to Eva-Maria Hedin, Anne Abrahamson, Agneta Roneus, and Ewa Larsson, Departments of Clinical Physiology and Radiology at the University of Uppsala, Sweden for their valuable support, and to Dr Louise Rose, for her helpful comments on the manuscript. Dr. Peter Herrmann, Department of Anaesthesiology, Emergency and Critical Care Medicine, University of Göttingen, Germany, provided the software (Maluna 2.04) and technical assistance for analyses of the CT scans. 
Authors contributions: Conception and design: LB, OM, GH, JB, AS, CS; Performing the experimental work: LB, OM, GH, CS; Analysis and interpretation: LB, OM, GH, JB, JT, AS, CS; Drafting the manuscript for important intellectual content: $\mathrm{LB}, \mathrm{OM}, \mathrm{GH}, \mathrm{JB}, \mathrm{JT}, \mathrm{AS}, \mathrm{CS}$

\begin{abstract}
Background: Endogenous pulmonary reflexes may protect the lungs during mechanical ventilation. We aimed to assess integration of continuous neurally adjusted ventilatory assist (cNAVA), delivering assist in proportion to diaphragm's electrical activity during inspiration and expiration, and Hering-Breuer inflation and deflation reflexes on lung recruitment, distension, and aeration before and after acute lung injury (ALI).
\end{abstract}

Methods: In 7 anesthetised rabbits with bilateral pneumothoraces, we identified adequate cNAVA level (cNAVA $\left.A_{A L}\right)$ at the plateau in peak ventilator pressure during titration procedures before (healthy lungs with endotracheal tube, $\left[\mathrm{HL}_{\mathrm{ETT}}\right]$ ) and after ALI (endotracheal tube $\left[A \mathrm{Al}_{\mathrm{ETT}}\right]$ and during non-invasive ventilation $\left.\left[A L I_{N I V}\right]\right)$. Following titration, CNAVA $_{A L}$ was maintained for 5 minutes. In 2 rabbits, procedures were repeated after vagotomy ( $\left.A L I_{E T T+V A G}\right)$. In 3 rabbits delivery of assist was temporarily modulated to provide assist on inspiration only. Computed tomography was performed before intubation, before ALI, during cNAVA titration, and after maintenance at $\mathrm{CNAVA}_{\mathrm{AL}}$.

Results: During $A L I_{E T T}$ and $A L I_{N I V}$, normally aerated lung-regions doubled and poorly aerated lung-regions decreased to less than a third $(p<0.05)$ compared to $\mathrm{HL}_{\mathrm{ETT}}$; no over-distension was 
observed. Tidal volumes were $<5 \mathrm{ml} / \mathrm{kg}$ throughout. Removing assist during expiration resulted in lung de-recruitment during $A L I_{E T T}$, but not during $A L I_{\text {NIV }}$. During $A L L_{E T T+V A G}$ the expiratory portion of EAdi disappeared, resulting in cyclic lung collapse and recruitment.

Conclusions: When using cNAVA in ALI, vagally mediated reflexes regulated lung recruitment preventing both lung over-distension and atelectasis. During non-invasive cNAVA the upper airway muscles play a role in preventing atelectasis. Future studies should be performed to compare these findings with conventional lung-protective approaches.

Abstract word count: 249 


\section{Introduction}

Continuous neurally adjusted ventilatory assist (cNAVA) is a new mode of mechanical ventilation. With cNAVA the electrical activity of the diaphragm (EAdi), reflecting the patient's neural respiratory drive, multiplied by an amplification factor (cNAVA level) is used to guide delivery of ventilatory pressure (Pvent) throughout the respiratory cycle, i.e. during inspiration and expiration. Hence, with cNAVA the patient's respiratory drive controls both, lung distension during inspiration and end-expiratory lung volume (EELV).

In 1868, Hering and Breuer described two reflexes, which could potentially play a role in lung protection during cNAVA: the "inspiratory-inhibiting reflex" and the "deflation-sensitive reflex" $^{\prime 1}$. These vagally-mediated reflexes sense lung stretch (and terminate inspiration), and lung deflation (and stimulate inspiration). ${ }^{2}$ Experimental and clinical studies suggest that intrinsic lung-protective reflexes are involved when using ventilator modes for which the subject's neural respiratory drive controls timing and magnitude of assist. ${ }^{3-11}$ Generally, the key findings pertain to a) down regulation of neural respiratory drive (reflected by EAdi) preventing increases in Pvent and tidal volume (Vt) when the proportionality constant (NAVA level) increases, b) presence of tonic EAdi activity throughout expiration in absence of positive endexpiratory pressure (PEEP), assumed to prevent lung-de-recruitment. However, no studies have specifically addressed if or how lung afferent reflexes may modulate neural respiratory drive to protect the lungs against over-distension and de-recruitment in acute lung injury (ALI). 
We used a rabbit model with open thorax and bilateral pneumothoraces. This model removed the contribution of the respiratory muscles and chest wall to lung inflation and deflation and prevented receptors sensing changes in lung volumes associated with the magnitude of ventilatory assist. Thus, feedback control of neural respiratory drive relied mainly on vagally mediated reflexes from the lungs.

We hypothesized that systematically increasing the cNAVA level from zero to high levels and performing computed tomography (CT) would allow us to observe the reflex-regulation of lung recruitment. Specifically, we assessed the effect of the Hering-Breuer inflation and deflation reflexes on lung recruitment, distension and aeration with cNAVA before and after ALI. We further hypothesized that temporarily removing ventilator assist during expiration would allow evaluating whether the animals maintain lung recruitment using either so-called "tonic EAdi" when intubated or expiratory flow limitation generated by the upper airways when noninvasively ventilated. Vagotomy served to confirm that the involved reflexes were vagally mediated.

Some of the results have been previously reported in abstract form. ${ }^{12}$ 


\section{Methods}

The study was performed according to the National Institutes of Health guidelines for the use of experimental animals and was approved by the University of Uppsala. Seven adult male New Zealand white rabbits weighing 3.1-3.9kg were studied.

\section{Sedation and instrumentation}

Bilateral cannulation of the ear veins and arteries was performed. Continuous sedation (ketamine-hydrochloride $40 \mathrm{mg} / \mathrm{kg} \cdot \mathrm{h}^{-1}$, xylazine $4 \mathrm{mg} / \mathrm{kg} \cdot \mathrm{h}^{-1}$ ) and Ringer's lactate solution $\left(5 \mathrm{ml} / \mathrm{kg}^{-1} \mathrm{~h}^{-1}\right)$ was infused. Transcutaneous oxygen saturation, arterial blood pressure, body temperature, and heart rate were monitored. EAdi was assessed with an $8 \mathrm{~F}$ oro-gastric catheter mounted with an array of 8 electrode pairs. Rabbits were ventilated in volume control ventilation (VCV) mode at $6 \mathrm{ml} / \mathrm{kg}$ with PEEP of $5 \mathrm{cmH}_{2} \mathrm{O}$ (Servo-I, Maquet Critical Care, Solna, Sweden).

\section{CNAVA}

cNAVA was performed with a custom made device (NeuroVent R\&D Inc., Toronto, Canada) that delivers pressure in proportion to the EAdi waveform throughout inspiration and expiration as previously described. ${ }^{13,14}$ The proportionality between pressure delivered and EAdi was adjusted by multiplying the EAdi by a gain factor, i.e. the cNAVA level (in $\mathrm{cm} \mathrm{H}_{2} \mathrm{O} / \mu \mathrm{V}$ ). 


\section{cNAVA titration}

For cNAVA titration, performed at various time-points (Figure 1), cNAVA level commenced at zero and was increased every 2 seconds by $0.08 \mathrm{cmH}_{2} \mathrm{O} / \mu \mathrm{V}$ (during non-invasive cNAVA increments were $0.32 \mathrm{cmH}_{2} \mathrm{O} / \mu \mathrm{V}$ ). The trend graphs for Pvent displayed a characteristic twophased response, i.e. a transition from an initial steep increase to a less steep increase. Adequate cNAVA level (cNAVA ${ }_{A L}$ ) was visually identified on Pvent trend graphs at the time-point where a plateau in peak ventilator pressure $\left(\right.$ Pvent $\left._{\max }\right)$ was reached., ${ }^{75-17}$ On completion of titration, ventilation continued for 5 minutes at CNAVA $A_{A L}$.

\section{Computed tomography}

Spiral CT images were acquired with a SOMATOM Sensation 16 (Siemens, Erlangen, Germany) with the following settings: $120 \mathrm{kVp}, 147 \mathrm{~mA}$, exposure time $500 \mathrm{~ms}, 3 \mathrm{~mm}$ slice collimination resulting in 33-39 single scans per spiral with a matrix of $512 \times 512$ voxels depending on lung dimensions.

\section{Protocol (Figure 1)}

A spiral CT scan was obtained with animals in supine position, sedated and spontaneously breathing, before intubation and induction of acute lung injury (ALI), (healthy lung control, $\left.H L_{C T R L}\right)$. 
Then animals were orally intubated (cuffed endotracheal tube (ETT), 3.5 I.D., Willy-Rusch $\mathrm{GmbH}$, Germany), the EAdi catheter was inserted, midline sternotomy and distention of the sternum was performed and bilateral pneumothoraces were induced. Spiral CT scans were performed during CNAVA titration, and after ventilation at CNAVA $A_{A L}$ for 5 minutes $\left(\mathrm{HL}_{\mathrm{ETT}}\right)$.

Then, acute lung injury (ALI) was induced. After neuromuscular paralysis (pancuroniumbromide $0.02 \mathrm{mg} / \mathrm{kg}$ ) in VCV mode $\left(6 \mathrm{ml} / \mathrm{kg}, \mathrm{PEEP}=5-7 \mathrm{cmH} \mathrm{H}_{2} \mathrm{O}\right), 2 \mathrm{~mL} / \mathrm{kg}$ hydrochloric acid $(\mathrm{pH}$ 1.5) was instilled into the trachea, followed by an inspiratory hold at $25 \mathrm{cmH}_{2} \mathrm{O}$ for ten seconds. The procedure was repeated after five minutes. After another 10 minutes on VCV, an ABG was obtained. When spontaneous breathing was restored, spiral CT scans were performed during cNAVA titration, and after 5 minutes at $\mathrm{CNAVA}_{\mathrm{AL}}\left(\mathrm{ALI}_{\mathrm{ETT}}\right)$.

Then, the oral ETT was removed and assist was delivered non-invasively using a single nasal prong (3.0 or 3.5 I.D., Blue-line, Portex, Kent, UK). Spiral CT scans were performed during cNAVA titration, and after 5 minutes of CNAVA $_{A L}\left(A L I_{N I V}\right)$.

\section{Removing assist during expiration}

In three animals, immediately following maintenance of $\mathrm{CNAVA}_{\mathrm{AL}}$ for 5 minutes we temporarily modulated assist, such that no pressure was applied during expiration (assist on inspiration only). This procedure was performed during both $A L I_{E T T}$ and $A L I_{N I V}$. Continuous CT scans of the same lung slice (static CT scans) were performed. 


\section{Vagotomy}

In two animals, tracheostomy was performed (4.0 I.D., Mallinckrodt Inc., St. Louis, USA) followed by bilateral vagotomy (VAG) at the neck level to eliminate vagal reflexes. ${ }^{15,18}$ Absence of the Hering-Breuer reflex was determined by distinct slowing of the respiratory rate. ${ }^{18}$ This part of the protocol was performed to confirm previous work showing that removal of vagal feedback after ALI in rabbits is associated with abolishment of tonic EAdi during expiration and hence cyclic lung de-recruitment. CT scans were performed during cNAVA titration and after 5 minutes at $c N A V A_{A L}\left(A L I_{E T T+V A G}\right)$.

\section{Data acquisition and analysis}

A single investigator blinded to experimental conditions analyzed all CT images (Maluna 2.04, University of Göttingen, Germany) as previously described ${ }^{19-21}$. Using standard thresholds for Hounsfield units ${ }^{20,22}$ we defined four degrees of aeration. Lung volume was calculated as the sum of gas volumes of all voxels that made up the lung volume in the spiral CT.

EAdi was acquired at a sampling rate of $2 \mathrm{kHz}$. Pvent and airflow were measured at the proximal end of either the ETT or the single nasal prong, and acquired at $100 \mathrm{~Hz}$. The EAdi and Pvent waveforms were characterized by the highest (EAdi $i_{\max }$ and Pvent $\mathrm{max}_{\max }$ ) and lowest (EAdi $\mathrm{Iin}_{\min }$ and Pvent $\left.t_{\min }\right)$ for each breath. Neural breathing frequency $\left(\mathrm{Bf}_{\text {neural }}\right)$ was calculated from the EAdi 
waveform. During the 5 minutes of $\mathrm{CNAVA}_{\mathrm{AL}}$, average values of these parameters were calculated in the final minute.

\section{Statistical analysis}

Statistical analysis was performed with SigmaStat ${ }^{\mathrm{TM}}$ (3.11, Systat Software Inc., San Jose, CA). We used Kolmogorov-Smirnov tests to assess data distribution. Paired data were compared with t-test or Wilcoxon Signed Rank test. HU distribution frequencies summed for the four attenuation compartments were compared between experimental conditions using Wilcoxon Signed Rank test. Level of significance was $p<0.05$. 


\section{Results}

All animals survived the protocol. Heart rate remained stable, systolic and diastolic blood pressures at experiment completion were $77 \pm 5$ and $55 \pm 5 \mathrm{mmHg}$, respectively. $A B G$ and breathing pattern values are given in Table 1 . Ten minutes after induction of $\mathrm{ALI}, \mathrm{PaO}_{2} / \mathrm{FiO}_{2}$ was reduced to $92 \pm 26$ ( $p<0.05$ vs $\mathrm{HL}_{\mathrm{ETT}}$ ). After 5 minutes at $C N A V A_{A L}, \mathrm{Vt}$ was slightly different when comparing $\mathrm{HL}_{\mathrm{ETT}}$ and $\mathrm{ALI}_{\mathrm{ETT}}(\mathrm{p}=0.022)\left(0.9 \mathrm{ml} / \mathrm{kg}\right.$ difference). $\mathrm{Bf}_{\text {neural }}$ increased after $\mathrm{ALI}(\mathrm{p}<0.001)$. $\mathrm{PaO}_{2} / \mathrm{FiO}_{2}$ was lower during $\mathrm{HL}_{\mathrm{ETT}}$ and $\mathrm{ALI}_{\mathrm{NIV}}$ compared to $\mathrm{ALI}_{\mathrm{ETT}}(\mathrm{p}<0.05)$, and $\mathrm{PaCO}_{2}$ was higher during all conditions compared to $\mathrm{HL}_{\mathrm{CTRL}}(\mathrm{p}<0.05)$.

Figure 2 shows the response of one intubated rabbit with ALI ( $A L I_{E T T}$ condition) to CNAVA titration. A video sequence of the same titration procedure is provided in the online supplement (Video 1).

Figure 3 shows group mean responses of EAdi $i_{\max }$ and EAdi $_{\min }$ (Panel A) as well as Pvent $\mathrm{max}_{\max }$ and Pvent $_{\text {min }}$ (Panel B) during cNAVA titration and after 5 minutes at cNAVA $A_{A L}$. During $\mathrm{HL}_{\mathrm{ETT}}, \mathrm{ALI}_{\mathrm{ETT}}$, and $A L I_{N I V}$, increasing cNAVA levels reduced $E A d i_{\max }$ and $E A d i_{\min }$ whereas Pvent max $_{\text {max }}$ and Pvent ${ }_{\min }$ increased initially and then plateaued. After five minutes at $\mathrm{CNAVA}_{\mathrm{AL}}$ there were further

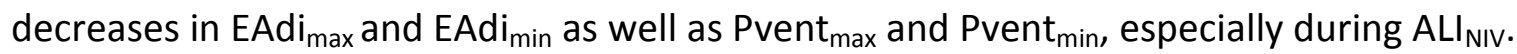

As depicted in Figure 4 and Table 2, lung aeration distribution after 5 minutes of cNAVA $A_{A L}$ was similar for $\mathrm{HL}_{\mathrm{CTRL}}$ and $\mathrm{HL}_{\mathrm{ETT}}$, comprising poor aeration (about $60 \%$ of lung tissue), normal 
aeration (about 30\%), and atelectatic lung tissue (about 10\%). Lung volumes were not different during $\mathrm{HL}_{\mathrm{CTRL}}$ and $\mathrm{HL}_{\mathrm{ETT}}(47.1 \pm 10.5 \mathrm{ml}$ and $38.2 \pm 6.5 \mathrm{ml}$, respectively) (Table 2$)$.

Following $A L I$ and regardless of interface $\left(A L I_{E T T}\right.$ or $\left.A L I_{N I V}\right)$, lung-regions classified as "normally aerated" increased to twice that observed during $\mathrm{HL}_{\mathrm{CTRL}}(p<0.05)$ and $\mathrm{HL}_{\mathrm{ETT}}(\mathrm{p}<0.05)$ after 5 minutes of cNAVA $A_{A L}$ (Figure 4). The contribution of lung regions classified as "poorly aerated" decreased to less than a third of the values observed during $\mathrm{HL}_{C T R L}(p<0.05)$ and $H L_{E T T}(p<0.05)$. Lung regions classified as atelectatic were not different from $\mathrm{HL}_{\mathrm{CTRL}}$ and $\mathrm{HL}_{\mathrm{ETT}}$. Lung volume increased significantly to $77.8 \pm 16.7 \mathrm{ml}$ and $65.3 \pm 17.5 \mathrm{ml}$ during $A L I_{E T T}$ and $A L I_{N I V}$ compared to both $\mathrm{HL}_{C T R L}(p<0.001, p=0.02)$ and $\mathrm{HL}_{E T T}(p<0.001, p=0.014)$.

\section{Removing assist during expiration}

Removal of expiratory assist with $A \mathrm{LI}_{\mathrm{ETT}}$, resulted in lung de-recruitment, increased $\mathrm{EAdi}_{\min }$, and

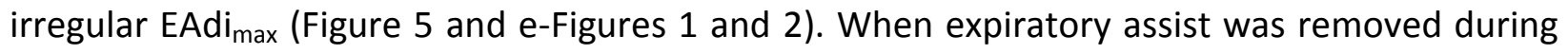
$\mathrm{ALI}_{\text {NIV }}$, two rabbits could maintain lungs fully recruited, with no change in $\mathrm{EAdi}_{\text {min }}$ and a stable $\mathrm{EAdi}_{\text {max }}$ and $\mathrm{Bf}_{\text {neural }}$ (Figure 5 and e-Figure 1). One rabbit maintained the same lung-recruitment during $A \mathrm{AL}_{\text {NIV }}$ as during $A \mathrm{AI}_{\mathrm{ETT}}$ but with half the $\mathrm{EAdi}_{\min }$ and irregular $\mathrm{EAdi}_{\max }$ (e-Figure 2).

\section{Vagotomy}

Bilateral vagotomy $\left(A L I_{E T T+V A G}\right)$ abolished $E_{A d i}$ and consequently Pvent min $_{\text {min }}$ compared to prevagotomy $\left(A L I_{E T T}\right)$ (Figure 6 and e-Figure 3). When increasing CNAVA levels during $A L I_{E T T+V A G}$, the 
decrease of EAdi $\mathrm{max}_{\max }$ was delayed (compared to $A \mathrm{LI}_{\mathrm{ETT}}$ ) causing Pvent $\mathrm{max}_{\max }$ to over-shoot before reaching a plateau. (Figure 6, panel B, bottom right and e-Figure 3). After 5 minutes at cNAVA $A_{A L}$ lungs were noticeably de-recruited (Panel C in Figure 6 and e-Figure 3). Lung volume decreased from $88.1 \mathrm{ml}$ and $79.9 \mathrm{ml}$ during $A \mathrm{AL}_{E T T}$ to $40.6 \mathrm{ml}$ and $45.8 \mathrm{ml}$ during $A \mathrm{AL}_{\mathrm{ETT}+V A G} . \mathrm{Bf}_{\text {neural }}$ decreased from $73 \mathrm{bpm}$ and $69 \mathrm{bpm}$ to $15 \mathrm{bpm}$ and $16 \mathrm{bpm}$, respectively.

Augmented neural inspiratory efforts (sighs) could be observed (Figure 6 and e-Figure 3). During CNAVA titration, sighs were observed in 4 of 7 rabbits during $\mathrm{HL}_{\mathrm{ET}}$ and in 6 of 7 rabbits during $A \mathrm{LI}_{\mathrm{ETT}}$. No sighs were observed in vagotomized rabbits.

Figure 7 depicts $C T$-images for inspiration and expiration in one animal during $H L_{E T T}, A L I_{E T T}$, $A L I_{N I V}$, and $A L I_{E T T+V A G}$. There is marked lung recruitment from $H L_{E T T}$ to both, $A L_{E T T}$ and $A L_{N I V}$. $A I_{E T T+V A G}$ resulted in lung de-recruitment and induced cycling between lung-collapse and recruitment. 


\section{Discussion}

Our study is unique and the first to demonstrate the functional impact of respiratory drive assessed as EAdi upon lung aeration, recruitment, and volume. We found that during ALI inherent, vagally-mediated reflexes favor a neural breathing pattern that promotes both lung aeration without over-distension and preservation of EELV.

More specifically, by using a ventilator that is neurally-controlled during inspiration and expiration, a model uncoupling the lungs from the respiratory muscles and chest wall, and sequential CT scans, we demonstrated that reflex responses to early experimental ALI involve: 1) an increase in diaphragm tonic activity which preserves EELV and prevents lung derecruitment; and 2) inhibition of peak inspiratory EAdi, thus limiting lung-distending pressure and preventing over-distension. Interestingly, despite these reflex changes, $\mathrm{Vt}$ after $\mathrm{ALI}$ remained similar or lower than before ALI. Bilateral vagotomy abolished these responses, supporting that the above-described protective mechanisms were mediated by vagal afferents. We also demonstrated that during non-invasive ventilation, lung de-recruitment is prevented, presumably via expiratory flow limitation by upper airway muscles.

\section{Preservation of EELV}

HCL-induced ALI promotes heterogeneous injury and de-recruitment of the lungs. ${ }^{23}$ During spontaneous breathing, maintenance of diaphragm activation during exhalation is a fundamental mechanism to prevent lung de-recruitment. ${ }^{24,25}$ Previous work in both intubated rabbits with experimental $A L I$ and in intubated children shows that tonic EAdi increases when 
PEEP is reduced. ${ }^{3,26}$ In the present study, EAdi ${ }_{\min }$ markedly increased when lungs collapsed after $\mathrm{ALI}$ at the start of titration (cNAVA levels = zero). EAdi $_{\min }$ then continuously decreased and lungs were recruited as Pvent $_{\min }$ (i.e. minimal pressure during exhalation restoring EELV) increased following continuously increasing cNAVA levels.

Instantaneous appearance of tonic EAdi induced by lung de-recruitment after removing assist during "neural expiration" provided evidence that tonic EAdi is reflex governed. Furthermore, loss of tonic EAdi after bilateral vagotomy resulting in cyclic expiratory collapse and inspiratory recruitment (an extremely injurious pattern of ventilation ${ }^{27}$ ) support that the reflex maintaining EELV is-was vagally mediated. The presence of tonic activity after ALI and its disappearance after bilateral vagotomy has been previously demonstrated in rabbits with $\mathrm{ALI}^{3,15}$ and assumed to affect lung-recruitment. ${ }^{26}$ The present study provides demonstrations in two rabbits how typical neural drive response to eliminated Hering-Breuer reflex influences lung-recruitment and breathing pattern. It should be noted that there was no possibility to control for non-lung effects of bilateral vagotomy at the neck.

Modulation of respiratory rate and expiratory time can also promote increases in EELV. ${ }^{28}$ The significant increase of respiratory rate we observed after ALI may have contributed to lung recruitment. Additionally, the impact of sighs on lung recruitment observed in our study is in agreement with previous observations in ARDS and ALI. ${ }^{29}$ 
Another possible mechanism for preventing lung deflation is upper airway muscles involvement, which may be activated dynamically during exhalation to brake expiratory flow. ${ }^{30}$ Beck et al previously demonstrated in rabbits with ALI that tonic EAdi disappeared when the endotracheal tube was removed. ${ }^{31}$ In the present study we demonstrated that lungs remained fully recruited without appearance of tonic EAdi when expiratory assist was removed during $\mathrm{ALI}_{\text {NIV. }}$ We hypothesize that the deflation-sensitive Hering-Breuer reflex was activated when the upper airway muscles were "blocked" by intubation and inactivated when upper airway modulation was present.

\section{Protection from lung over-distension}

We also found reflex-prevention of lung over-distension during cNAVA titration (with intact

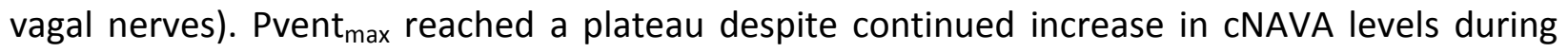
both $A L I_{E T T}$ and $A L I_{N I V}$ CNAVA. Similar findings of EAdi down-regulation and lung-distending "pressure-ceiling" have previously been reported for transpulmonary pressures in rabbits with

$\mathrm{ALI}^{3}$ and during loaded breathing. ${ }^{15}$ Several patient studies demonstrated plateaus in Vt, Pvent, or transpulmonary pressure during increasing NAVA levels associated with EAdi suppression. $^{7,9,10,16,17,33,34}$

Our observation of a more favourable distribution of aeration with less atelectatic regions after ALI compared to normal lungs (i.e. HCL induced lung injury improved pattern of lung aeration in cNAVA ventilated rabbits) agree with findings that $\mathrm{HCL}$-induced $\mathrm{ALI}$ induces heterogeneous injury and de-recruitment of the lungs. ${ }^{23}$ This is in stark contrast to healthy homogenous lungs 
which are easily recruited. ${ }^{35}$ Our findings of a major shift to normally aerated and not overdistended lung-regions after ALI is in agreement with lung aeration observed using current clinical standards e.g. so-called open-lung concept using assist-controlled ventilation at $6 \mathrm{ml} / \mathrm{kg}$ predicted body weight with high PEEP (e.g. PEEP set at $2 \mathrm{cmH}_{2} \mathrm{O}$ above lower inflection point of pressure-volume curve). ${ }^{36}$

\section{Method critique}

To demonstrate lung-protective reflexes, muscle force, fatigue, and feedback should not be interfering factors. In our model, the respiratory muscles and chest wall mechanics played no role in ventilation, nor in lung recruitment. Using cNAVA we replaced the respiratory muscles with a "non-fatigable" neurally driven pressure delivery device that is efficient in generating pressure regardless of lung-volumes. Thus, any influence of respiratory muscle activity (e.g. weakness due to hyperinflation) was abolished. Additionally, while maintaining the efferent neural activation to the respiratory muscles, an open thorax and exposed lungs eliminated the passive outward recoil of the ribcage and diaphragm, which governs the FRC. EAdi allowed the diaphragm to contract, however its ability to generate pressure and distend the lungs was disabled. Also, the afferent feedback from the respiratory muscles was abolished due to inability to sense changes in ventilatory assist and lung volumes.

In our open chest model, pressure delivered with cNAVA closely reflected transpulmonary pressure (except when cNAVA was delivered non-invasively due to large pressure loss associated with leaks). Hence, the ventilator replaced the mechanical "pump-function" of the 
"disabled" respiratory muscles. cNAVA was controlled by EAdi, which responds to the same neural output as the respiratory muscles when functioning.

Our study has some limitations. Animals were unattended in the CT scanner, and thus may have received more sedation than usual. This may explain the higher $\mathrm{PaCO}_{2}$ levels in our study compared to other studies ${ }^{5,31}$ and the poorly aerated lungs before ALI. ${ }^{37}$ It should be noted that the "open chest model" eliminates the "normal" intrapleural negative pressure recruitment of the lungs seen with an intact thorax, which could have influenced blood gases. However, despite the deep sedation level and open chest model, a strong lung-protective response could still be demonstrated after ALI.

\section{Conclusion}

This is the first study using an in vivo model to describe and quantify reflex-mediated regulation of lung recruitment and $\mathrm{Vt}$ in response to experimental $\mathrm{ALI}$. The reflex-mediated response was characterized by remarkable and homogenous lung-recruitment, prevention of atelectasis, and breathing with low Vt without over-distending the lungs. Our data also provide evidence that, after extubation, the upper airway muscles play a direct role in preventing atelectasis during non-invasive ventilation. Future studies should address if integration of ventilatory assist with neural reflexes may act to prevent VILI in a similar fashion to conventional lung-protective approaches, which aim to maintain an open lung and minimize Vt. 


\section{References}

1 Hering E, Breuer J. Die Selbststeurung der Athmung durch den nervus vagus. Sitzber Deut Akad Wiss Wien. 1868;57:672-7.

2 Widdicombe J. Reflexes from the lungs and airways: historical perspective. J Appl Physiol. 2006;101(2):628-34.

3 Allo JC, Beck JC, Brander L, Brunet F, Slutsky AS, Sinderby CA. Influence of neurally adjusted ventilatory assist and positive end-expiratory pressure on breathing pattern in rabbits with acute lung injury. Crit Care Med. 2006;34(12):2997-3004.

4 Sinderby C, Beck J, Spahija J, et al. Inspiratory muscle unloading by neurally adjusted ventilatory assist during maximal inspiratory efforts in healthy subjects. Chest. $2007 ; 131(3): 711-7$

5 Brander L, Sinderby C, Lecomte F, et al. Neurally adjusted ventilatory assist decreases ventilator-induced lung injury and non-pulmonary organ dysfunction in rabbits with acute lung injury. Intensive Care Med. 2009;35(11):1979-89.

6 Mirabella L, Grasselli G, Haitsma J, et al. Lung protection during non-invasive synchronized assist versus volume control in rabbits. Crit Care. 2014;18(1):R22.

7 Brander L, Leong-Poi $\mathrm{H}$, Beck J, et al. Titration and implementation of neurally adjusted ventilatory assist in critically ill patients. Chest. 2009;135(3):695-703. 
8 Piastra M, De Luca D, Costa R, et al. Neurally adjusted ventilatory assist vs pressure support ventilation in infants recovering from severe acute respiratory distress syndrome: Nested study. J Crit Care. 2014;29(2):312.e1-5.

9 Barwing J, Linden N, Ambold M, Quintel M, Moerer O. Neurally adjusted ventilatory assist vs. pressure support ventilation in critically ill patients: an observational study. Acta Anaesthesiol Scand. 2011;55(10):1261-71.

10 Firestone KS, Fisher S, Reddy S, White DB, Stein HM. Effect of changing NAVA levels on peak inspiratory pressures and electrical activity of the diaphragm in premature neonates. $J$ Perinatol. 2015;35(8):612-6.

11 Doorduin J, Sinderby CA, Beck J, van der Hoeven JG, Heunks LM. Assisted ventilation in patients with acute respiratory distress yyndrome: Lung-distending pressure and patientventilator interaction. Anesthesiology. 2015; 23(1):181-90.

12 Brander L, Moerer O, Beck J, et al. Neural control of lung recruitment and tidal volume (VT) in rabbits with acute lung injury (ALI) [abstract]. Am J Resp Crit Care Med. 2007;175:A94.

13 Sinderby C, Navalesi P, Beck J, Skrobik Y, Comtois N, Friberg S, Gottfried SB, Lindström L. Neural control of mechanical ventilation in respiratory failure. Nature Med. 1999;5(12):1433-6.

14 Liu L, Takahashi D, Qui H, Slutsky AS, Sinderby C, Beck J. Feasibility of neurally adjusted positive end-expiratory pressure in rabbits with early experimental lung injury. $B M C$ Anesthesiol. 2015;15(1):124. 
15 Lecomte F, Brander L, Jalde F, et al. Physiological response to increasing levels of neurally adjusted ventilatory assist (NAVA). Respir Physiol Neurobiol. 2009;166(2):117-24.

16 Tuchscherer D, Z'Graggen WJ, Passath C, Takala J, Sinderby C, Brander L. Neurally adjusted ventilatory assist in patients with critical illness-associated polyneuromyopathy. Intensive Care Med. 2011;37(12):1951-61.

17 Passath C, Takala J, Tuchscherer D, Jakob SM, Sinderby C, Brander L. Physiologic response to changing positive end-expiratory pressure during neutrally adjusted ventilatory assist in sedated, critically ill adults. Chest. 2010;138(3):578-87.

18 Moore RL. A study of the Hering-Breuer reflex. J Exp Med. 1927;46(5):819-37.

19 Zinserling J, Wrigge $\mathrm{H}$, Neumann $\mathrm{P}$, et al. Methodologic aspects of attenuation distributions from static and dynamic thoracic CT techniques in experimental acute lung injury. Chest. 2005;128(4):2963-70.

20 Gattinoni L, Caironi P, Pelosi P, Goodman LR. What has computed tomography taught us about the acute respiratory distress syndrome? Am J Respir Crit Care Med. 2001;164(9):1701-11.

21 Gattinoni L, Caironi $\mathrm{P}$, Cressoni $\mathrm{M}$, et al. Lung recruitment in patients with the acute respiratory distress syndrome. N Engl J Med. 2006;354(17):1775-86.

22 Hounsfield GN. Computerized transverse axial scanning (tomography). 1. Description of system. Br J Radiol. 1973;46:1016-22. 
23 Greenfield L, Singleton RP, McCaffree DR, Coalson JJ. Pulmonary effects of experimental graded aspiration of hydrochloric acid. Ann Surg. 1969;170(1):74-86.

24 Putensen C, Mutz NJ, Putensen-Himmer G, Zinserling J. Spontaneous breathing during ventilatory support improves ventilation-perfusion distributions in patients with acute respiratory distress syndrome. Am J Respir Crit Care Med. 1999;159(4):1241-8.

25 Wrigge $\mathrm{H}$, Zinserling J, Neumann $\mathrm{P}$, et al. Spontaneous breathing improves lung aeration in oleic acid-induced lung injury. Anesthesiology. 2003;99(2):376-84.

26 Emeriaud G, Beck J, Tucci M, Lacroix J, Sinderby C. Diaphragm electrical activity during expiration in mechanically ventilated infants. Pediatr Res. 2006;59(5):705-10.

27 Tremblay L, Valenza F, Ribeiro SP, Li J, Slutsky AS. Injurious ventilatory strategies increase cytokines and c-fos m-RNA expression in an isolated rat lung model. J Clin Invest. 1997;99(5):944-52.

28 Syring RS, Otto CM, Spivack RE, Markstaller K, Baumgardner JE. Maintenance of endexpiratory recruitment with increased respiratory rate after saline-lavage lung injury. J Appl Physiol. 2007;102(1):331-9.

29 Hess DR, Bigatello LM. Lung recruitment: the role of recruitment maneuvers. Respir Care. 2002;47(3):308-17.

30 Kosch PC, Stark AR. Dynamic maintenance of end-expiratory lung volume in full-term infants. J Appl Physiol Respir Environ Exerc Physiol. 1984;57(4):1126-33. 
31 Beck J, Brander L, Slutsky AS, Reilly MC, Dunn MS, Sinderby C. Non-invasive neurally adjusted ventilatory assist in rabbits with acute lung injury. Intensive Care Med. 2008;34(2):316-23.

32 Grasselli G, Beck J, Mirabella L, Pesenti A, Slutsky AS, Sinderby C. Assessment of patientventilator breath contribution during neurally adjusted ventilatory assist. Intensive Care Med. 2012;38(7):1224-32.

33 Terzi N, Pelieu I, Guittet L, et al. Neurally adjusted ventilatory assist in patients recovering spontaneous breathing after acute respiratory distress syndrome: physiological evaluation. Crit Care Med. 2010;38(9):1830-7.

34 Patroniti N, Bellani G, Saccavino E, et al. Respiratory pattern during neurally adjusted ventilatory assist in acute respiratory failure patients. Intensive Care Med. 2012;38(2):230-9.

35 Rothen HU, Neumann P, Berglund JE, Valtysson J, Magnusson A, Hedenstierna G. Dynamics of re-expansion of atelectasis during general anaesthesia. Br J Anaesth. 1999;82(4):551-6.

36 Caironi P, Cressoni M, Chiumello D, Ranieri M, Quintel M, Russo SG, Cornejo R, Bugedo G, Carlesso E, Russo R, Caspani L, Gattinoni L. Lung opening and closing during ventilation of acute respiratory distress syndrome. Am J Respir Crit Care Med. 2010;181(6):578-86.

37 Rothen HU, Sporre B, Engberg G, Wegenius G, Hedenstierna G. Airway closure, atelectasis and gas exchange during general anaesthesia. Br J Anaesth. 1998;81(5):681-6. 


\section{Legends to Figures in the main text}

\section{Figure 1. Steps performed in the protocol}

From top to bottom: Order of the protocol in seven sedated animals undergoing instrumentation, intubation, and thoracotomy (before and after acute lung injury), extubation and non-invasive ventilation. At each protocol step, animals underwent CT scans during CNAVA level titrations. $\mathrm{HL}_{\mathrm{CTRL}}=$ healthy lung control; $\mathrm{HL}_{\mathrm{ETT}}=$ healthy lung with endo-tracheal tube; $A L I_{E T T}=$ after acute lung injury with endotracheal tube; $A L I_{N I V}=$ after acute lung injury and noninvasive ventilation with nasal prong; $A \mathrm{LI}_{\mathrm{ETT}+\mathrm{VAG}}=$ after acute lung injury with endotracheal tube and bilateral vagotomy. $\mathrm{cNAVA}_{\mathrm{AL}}=$ adequate $\mathrm{cNAVA}$ level (see Methods for description).

Figure 2. cNAVA level titration in an animal after acute lung injury $\left(A L I_{E T T}\right)$.

Panel A: Top to bottom: Values for lung volume, electrical activity of the diaphragm (EAdi), and pressure delivered by the ventilator (Pvent) are plotted versus cNAVA level and time.

Panel B: Each CT-scan image in panel B corresponds to the times indicated by shaded columns 1-6 in panel $A$. The shaded columns denoted $4 a-b$ and $5 a-b$ in panel $A$ and the corresponding CT-scans in panel $B$ demonstrate the effect of spontaneous sighs on lung recruitment.

At cNAVA = zero, Pvent and volume were zero, EAdi was high with relatively small, breathrelated swings that never returned to zero (so-called "tonic EAdi") (Panel A) and the lungs were collapsed (Panel B, image 1). Linear increases of the cNAVA level initially increased Pvent and lung volumes as well as their swings. EAdi decreased and breath-related EAdi swings (so called "phasic EAdi") appeared, resulting in lung recruitment (Panel B, images 2-4).

After about 40 seconds, both tonic and phasic EAdi decreased with increasing cNAVA level. Pvent and breath-related pressure swings plateaued and remained unchanged despite an almost threefold continued increase in the cNAVA level. Volume plateaued but increased in a stepwise fashion after spontaneous sighs by the animal. Spontaneous sighs produced significant increases in lung-recruitment (Panel $B$, images $4 a-b$ and $5 a-b$ ) until the lungs occupied almost the entire thoracic cavity (Panel $B$, image 6).

A video sequence of the same titration procedure is provided in the online supplement (Video 1). 
Figure 3. Group mean responses of electrical activity of the diaphragm (EAdi), and pressure delivered by the ventilator (Pvent) during cNAVA titrations.

Responses of EAdi max $_{\text {(solid symbols) and EAdi }}$ min (empty symbols) (Panel A) as well as Pvent max $_{\text {max }}$

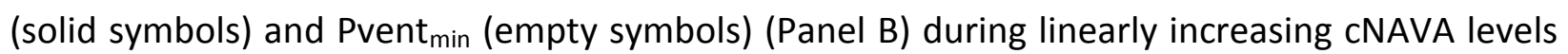
and after 5 minutes of ventilation at $\mathrm{CNAVA}_{\mathrm{AL}}$.

From top to bottom, group mean values obtained with i) healthy lungs and orally inserted endotracheal tube (ETT) (HLETT), ii) ALI with orally inserted ET-tube ( $\left.A L I_{E T T}\right)$, and iii) ALI with noninvasive ventilation ( $\left.A L I_{\text {NIV }}\right)$.

Values presented are mean $\pm S E M$. All comparisons at 5 minutes versus inflection point are significant, $p<0.05$, unless indicated: NS = no significant difference from $c N A V A_{A L}$ compared to inflection point value.

Figure 4. Distribution of lung aeration after 5 minutes of ventilation at the adequate cNAVA level (cNAVA $\left.A_{A L}\right)$

Frequency distribution of lung aeration during: $i)$ healthy lungs before intubation $\left(H L_{C T R L}\right)$, ii) healthy lungs and orally inserted endotracheal tube (ETT) (HLETT), iii) ALI with orally inserted ETtube ( $\left.A L I_{E T T}\right)$, and iv) $A L I$ with non-invasive ventilation ( $A L I_{N I V}$ ). Aeration listed at top. Data presented for group mean with SD.

Figure 5. CT-images and continuous time plots of electrical activity of the diaphragm (EAdi), and pressure delivered by the ventilator (Pvent) in one animal when expiratory assist is removed

During intubated conditions ( $\left.A \mathrm{LI}_{\mathrm{ETT}}\right)$, removal of expiratory assist (vertical arrow), resulted in elevated tonic EAdi, with minimally discernable phasic EAdi. Corresponding CT image indicates lung de-recruitment. When ventilated non-invasively $\left(A L I_{N I V}\right)$, removal of expiratory assist did not result in lung de-recruitment, and tonic EAdi did not increase, indicating that the upper airway muscles maintained the lungs recruited. See also e-Figures 1 and 2.

Figure 6. Responses of electrical activity of the diaphragm (EAdi), and pressure delivered by the ventilator (Pvent) during cNAVA titration and lung aeration in one animal with bilateral vagotomy 


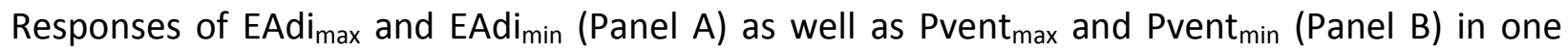
rabbit during linearly increasing cNAVA level and after 5 minutes of ventilation at CNAVA AL $_{\text {. }}$ Panel $\mathrm{C}$ shows distribution of lung aeration after 5 minutes of ventilation at CNAVA $A$ L. Top panels show values obtained during $A L I$ with orally inserted ET-tube ( $\left.A L L_{E T T}\right)$ and bottom panels show values obtained during $A L I$ and bilateral vagotomy with ET-tube via tracheostomy ( $\left.A L I_{E T T+V A G}\right)$. See also e-Figure 3.

\section{Figure 7. CT-images obtained in one animal under different experimental conditions}

CT-images for inspiration and expiration in one animal during $\mathrm{A}$ ) healthy lungs and orally inserted ET-tube $\left.\left(H L_{E T T}\right), B\right) A L I$ with orally inserted ET-tube (ALIETT), C) ALI with non-invasive ventilation ( $A L I_{N I V}$ ) and $\left.D\right) A L I$ and bilateral vagotomy with ET-tube via tracheostomy $\left(A L I_{E T T+V A G}\right)$. 
Animal preparation (sedation, instrumentation)

spiral CT scan

Healthy Lungs

No assist

$\mathrm{HL}_{\mathrm{CTRL}}$

Oral endo-tracheal tube placement
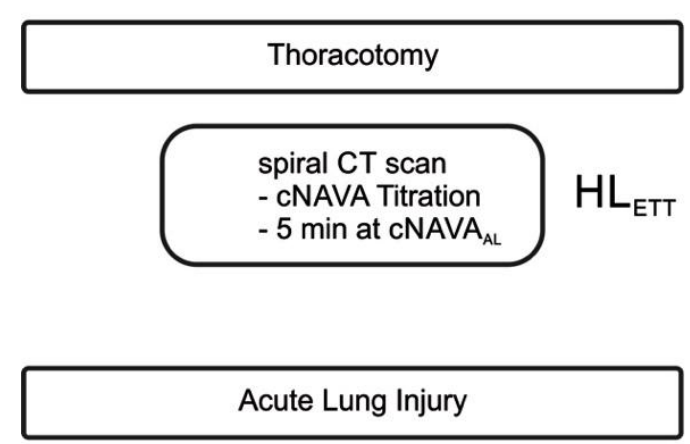

- cNAVA Titration

- 5 min at cNAVA

$A L I_{E T T}$

\section{$N=3 \sqrt{\text { cNAVA }}$}

no expiratory assist

static CT scan

Extubate and insert nasal prong

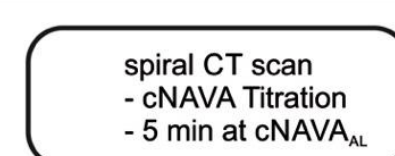

$N=2$

Tracheotomy + vagotomy

static CT scan

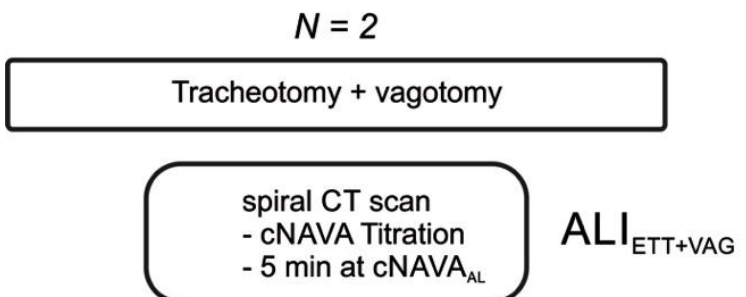

Figure 1 
A

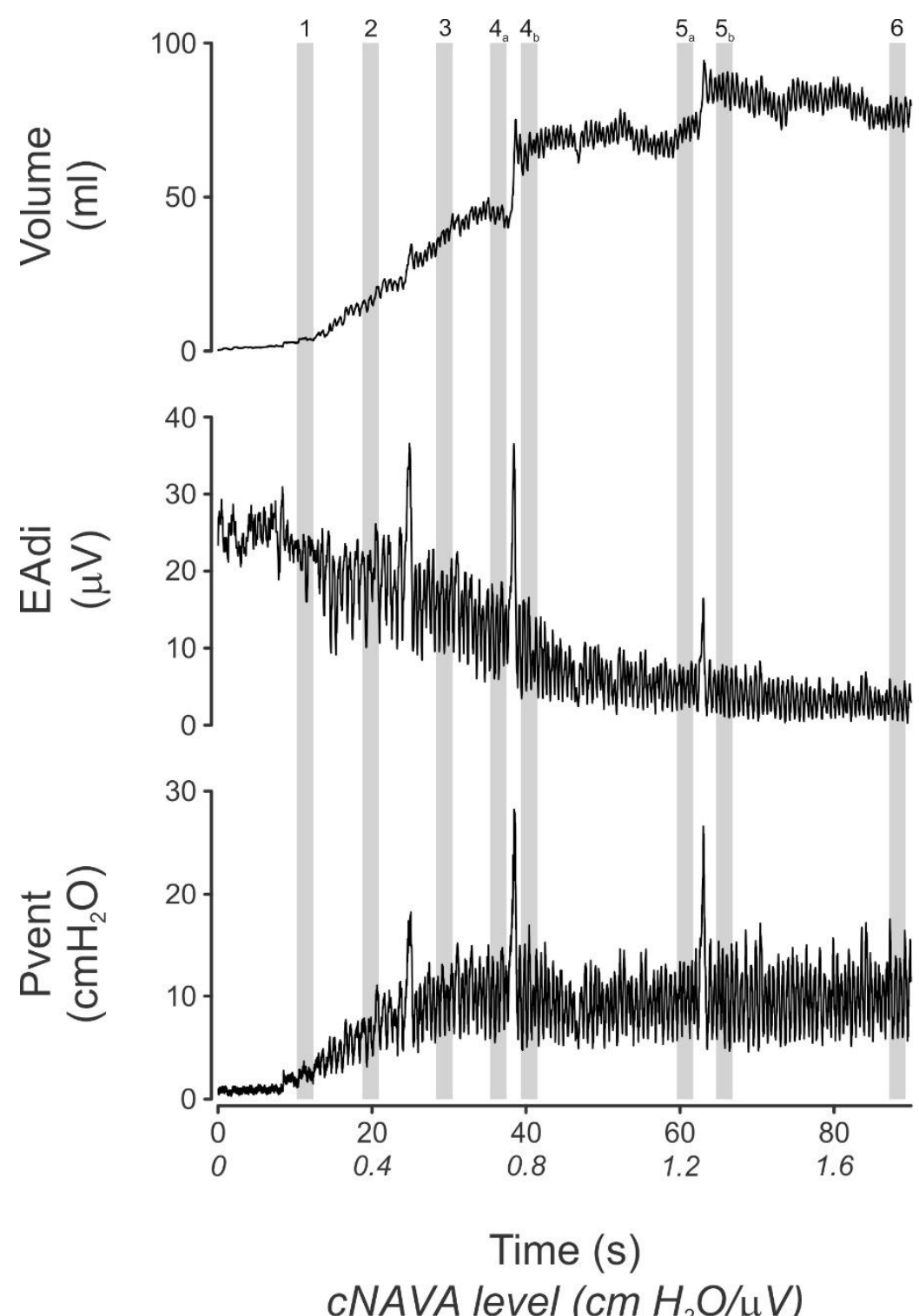

B
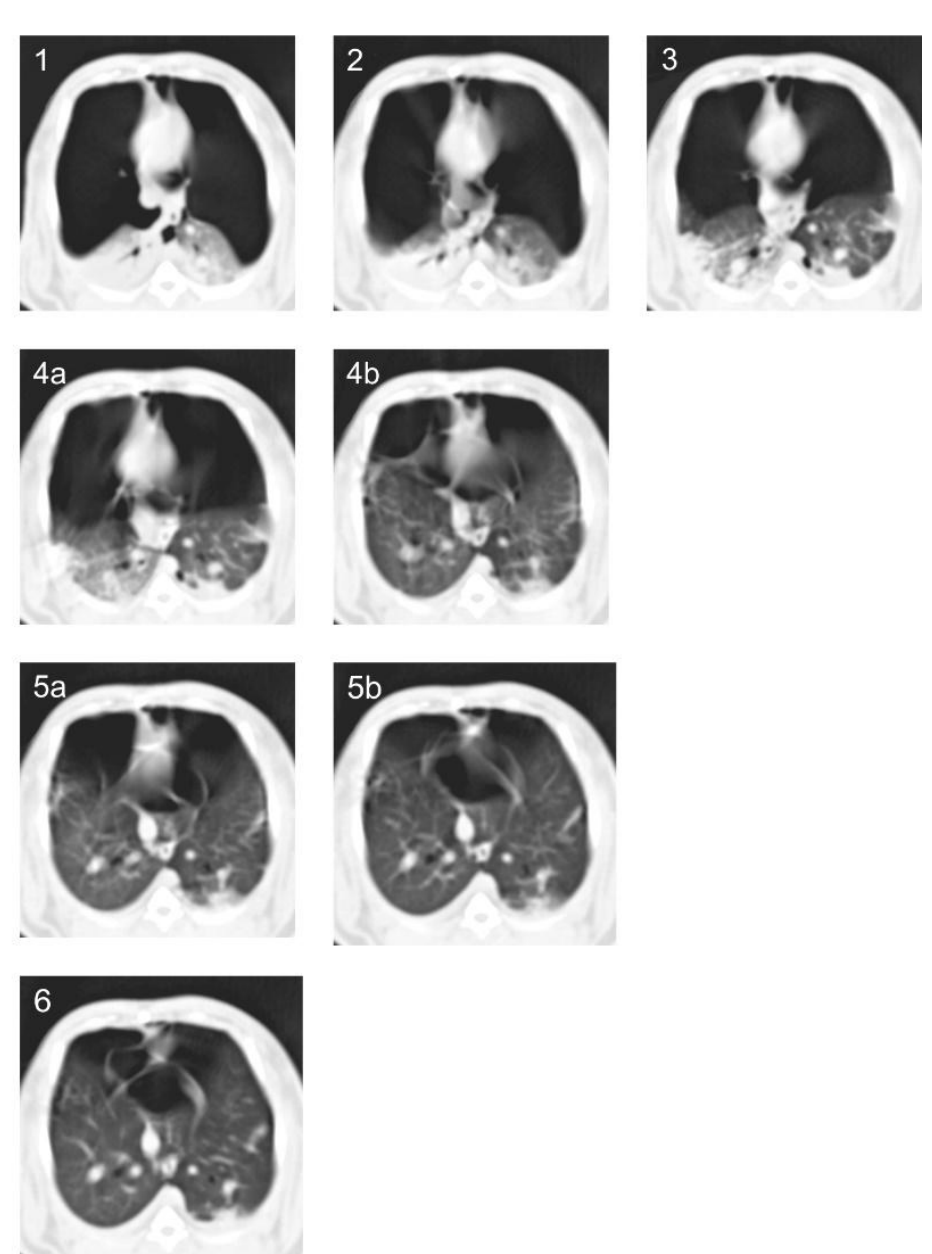

Figure 2 

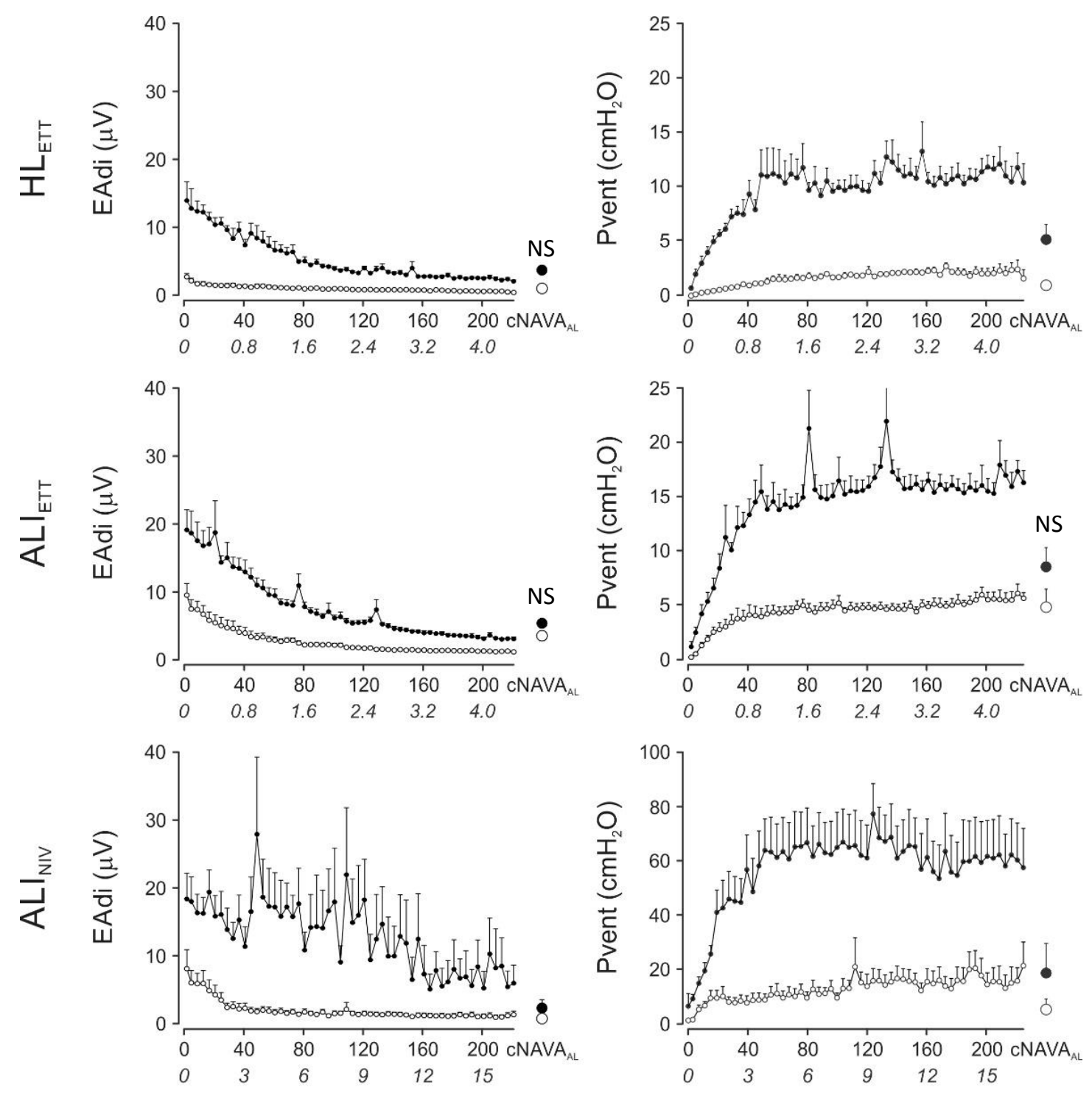

Time $(\mathrm{s})$
cNAVA level $\left(\mathrm{cm} \mathrm{H}_{2} \mathrm{O} / \mu \mathrm{V}\right)$

\author{
Time (s) \\ cNAVA level $\left(\mathrm{cm} \mathrm{H}_{2} \mathrm{O} / \mu \mathrm{V}\right)$
}

Figure 3 

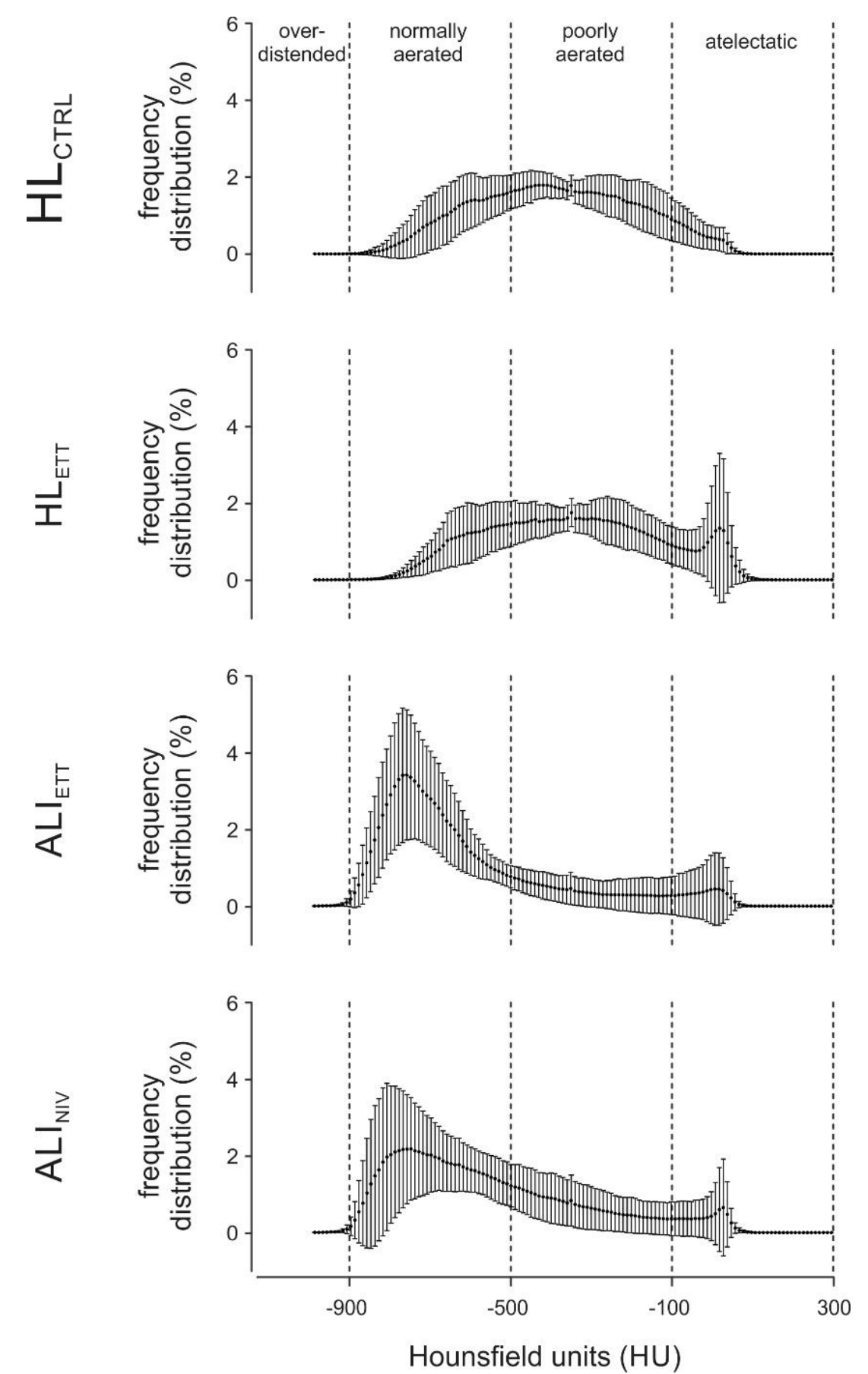

Figure 4 


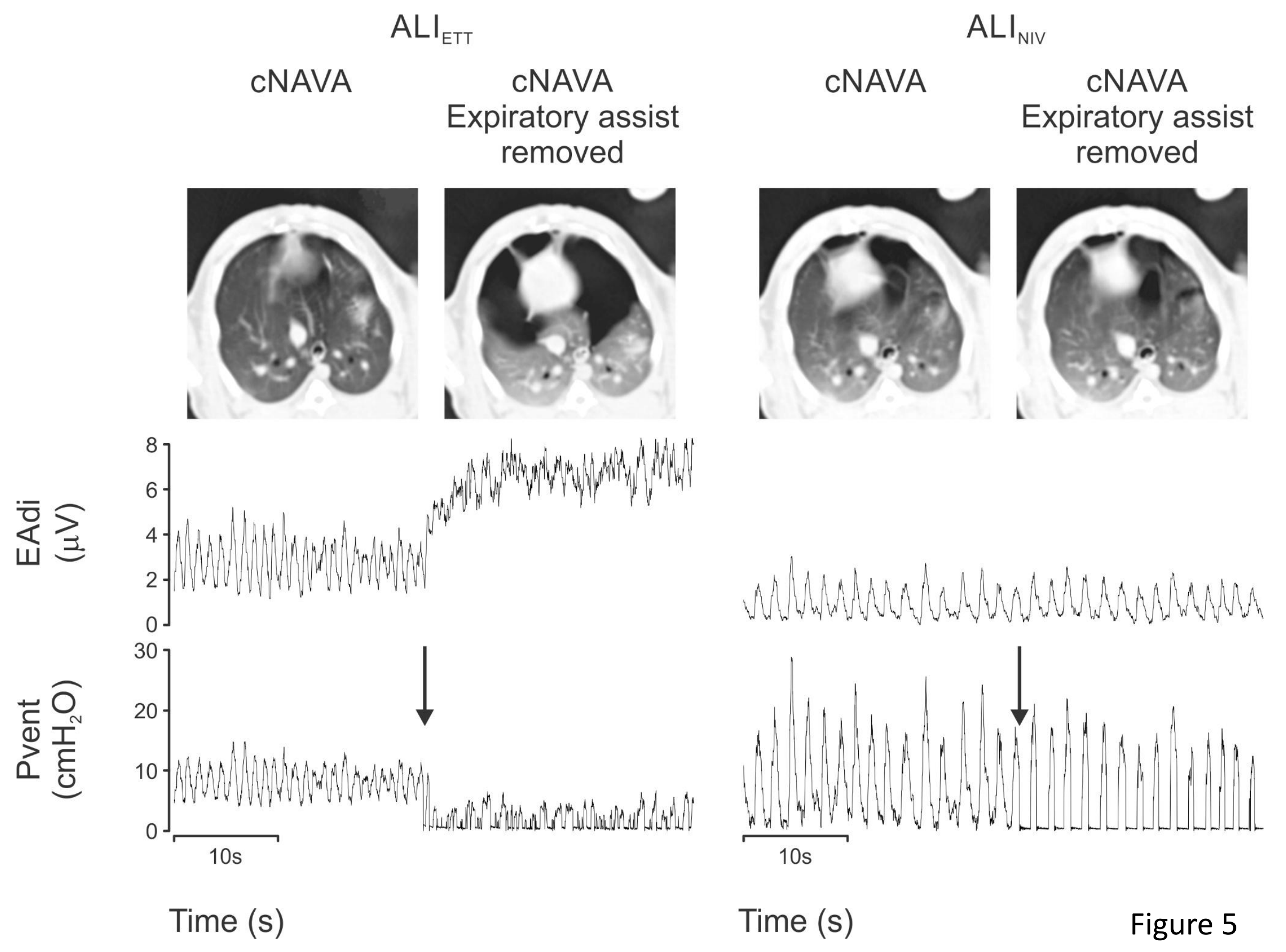


A

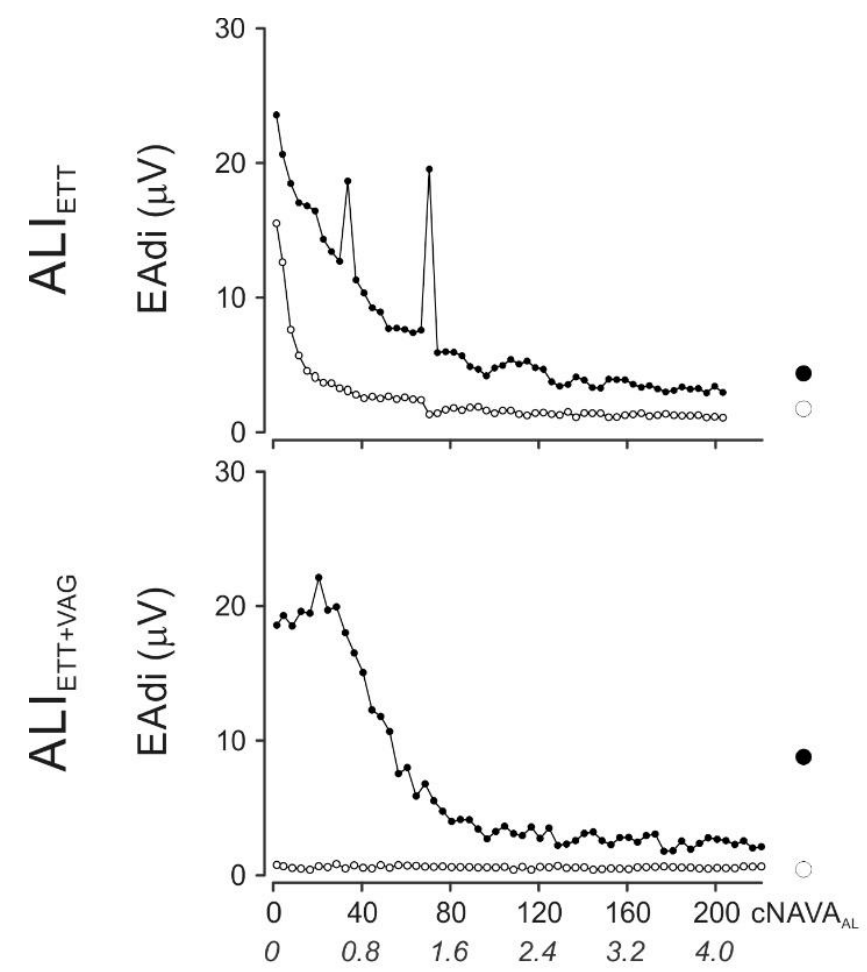

Time (s)

cNAVA level $\left(\mathrm{cm} \mathrm{H}_{2} \mathrm{O} / \mu \mathrm{V}\right)$
B
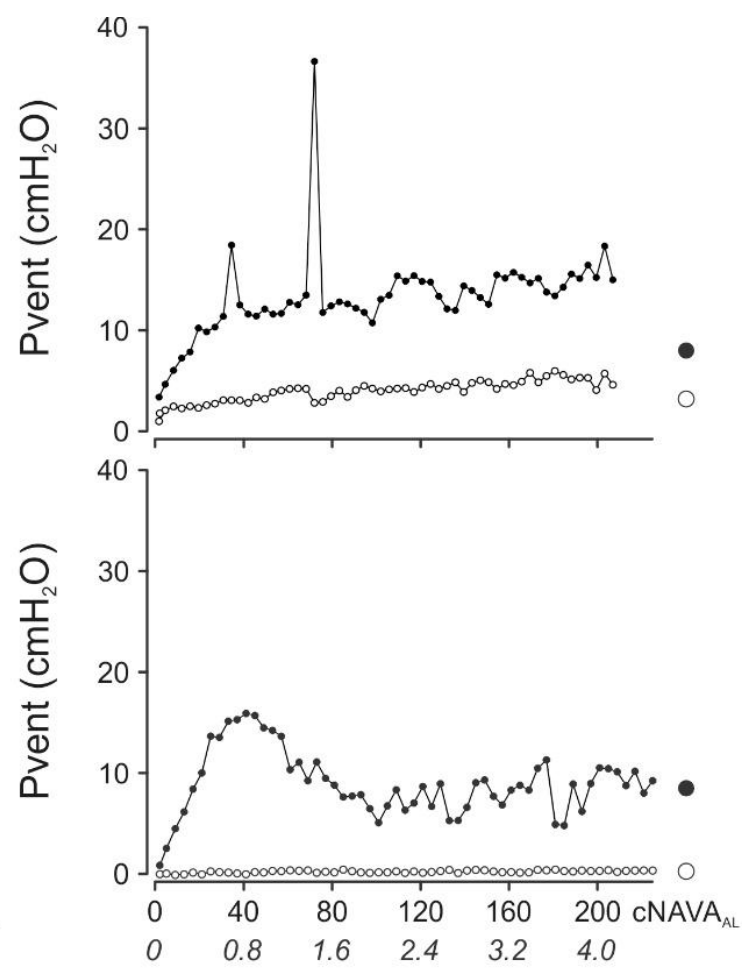

Time (s)

cNAVA level $\left(\mathrm{cm} \mathrm{H}_{2} \mathrm{O} / \mu \mathrm{V}\right)$
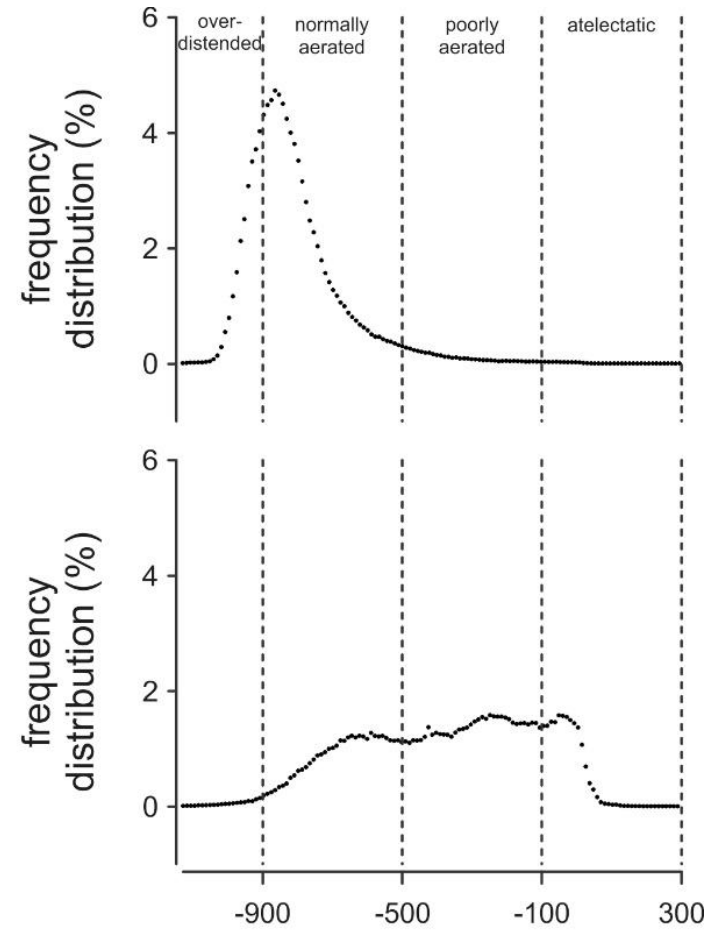

Hounsfield units (HU)

Figure 6 


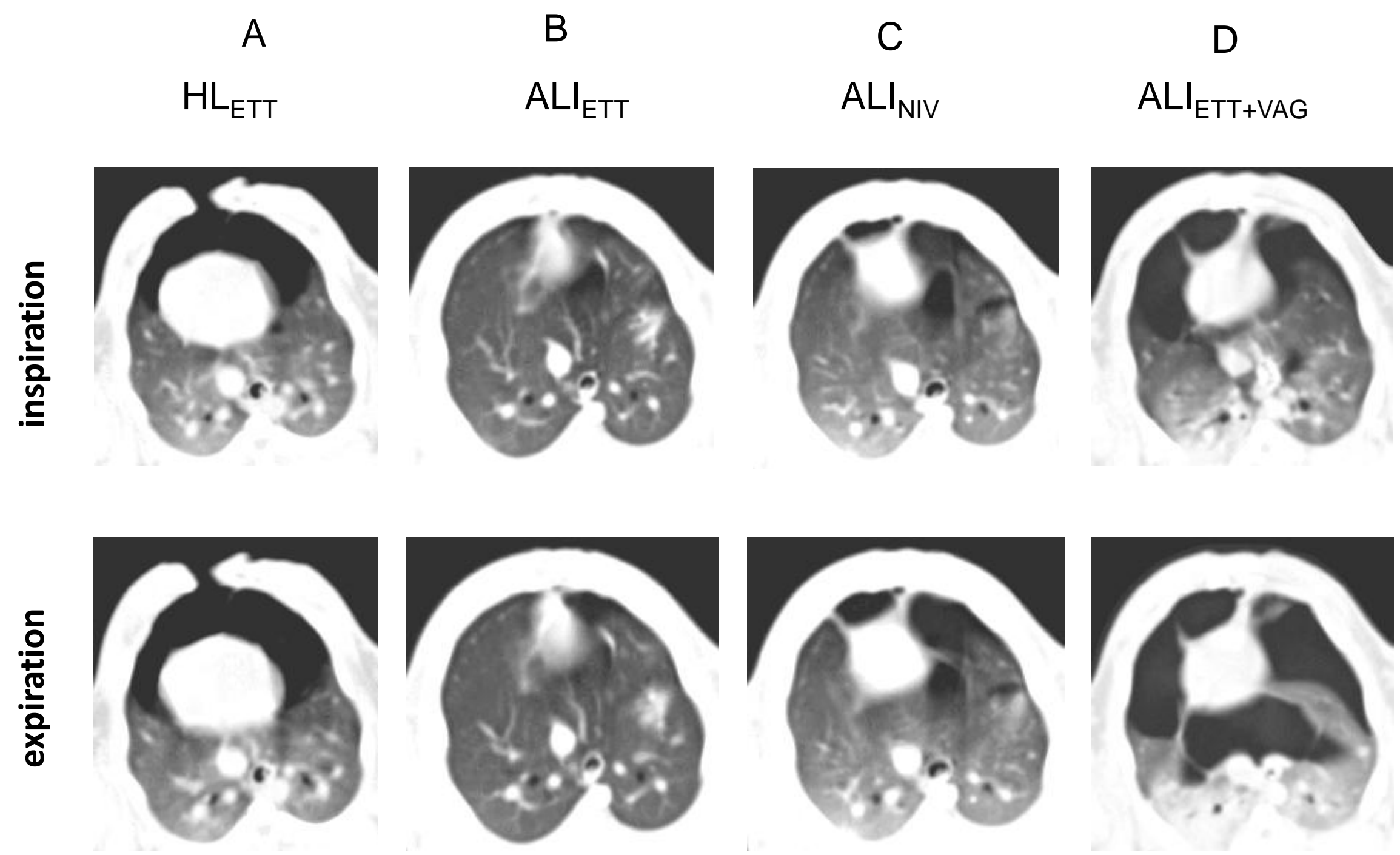

FIGURE 7 


\begin{tabular}{|l|l|l|l|l|}
\hline & $\mathrm{HL}_{\mathrm{CTRL}}$ & $\mathrm{HL}_{\text {ETT }}$ & $\mathrm{ALI}$ \\
\hline $\mathrm{V}_{\mathrm{T}}(\mathrm{ml} / \mathrm{kg})$ & & $3.8 \pm 0.6$ & $4.7 \pm 0.9^{\mathrm{b}}$ & $\mathrm{ALI}$ NIV \\
\hline $\mathrm{Bf}_{\text {neural }}(\mathrm{bpm})$ & & $42 \pm 9$ & $72 \pm 12^{\mathrm{b}}$ & $52 \pm 10^{\mathrm{c}}$ \\
\hline $\mathrm{SaO}_{2}(\%)$ & $95.0 \pm 7.0$ & $92.5 \pm 4.2$ & $98.1 \pm 1.3$ & $96.6 \pm 3.9$ \\
\hline $\mathrm{PaO}_{2} / \mathrm{FiO}_{2}$ & $420 \pm 129$ & $254 \pm 101^{\mathrm{a}}$ & $342 \pm 137$ & $245 \pm 132^{\mathrm{a}}$ \\
\hline $\mathrm{PaCO}_{2}(\mathrm{~mm} \mathrm{Hg})$ & $50.7 \pm 11.0$ & $72.3 \pm 15.2^{\mathrm{a}}$ & $75.3 \pm 10.0^{\mathrm{a}}$ & $79.4 \pm 12.2^{\mathrm{a}}$ \\
\hline $\mathrm{pH}$ & $7.36 \pm 0.08$ & $7.28 \pm 0.06^{\mathrm{a}}$ & $7.26 \pm 0.05^{\mathrm{a}}$ & $7.23 \pm 0.06^{\mathrm{a}}$ \\
\hline $\mathrm{HR}$ (per min) & $177 \pm 19$ & $173 \pm 10$ & $178 \pm 11$ & $176 \pm 8$ \\
\hline
\end{tabular}

Table 1: Group mean breathing pattern, vital signs and blood gas values assessed after 5 minutes on $\mathrm{CNAVA}_{\mathrm{AL}}$ during healthy lung conditions and after acute-lung injury conditions. $\mathrm{HL}_{C T R L}=$ healthy lung control; $\mathrm{HL}_{\mathrm{ETT}}$ = healthy lung with endo-tracheal tube; $\mathrm{ALI}_{\mathrm{ETT}}=$ after acute lung injury with endotracheal tube; $A L_{N I V}=$ after acute lung injury and non-invasive ventilation with nasal prong. $\mathrm{V}_{\mathrm{T}}=$ tidal volume; $\mathrm{Bf}_{\text {neural }}=$ neural breathing frequency; $\mathrm{HR}=$ heart rate.

Values presented are mean $\pm \mathrm{SD} .{ }^{a}$ indicates $p<0.05$ vs HL $\mathrm{CTRL}{ }^{\mathrm{b}}$ indicates $p<0.05 \mathrm{HL}_{\mathrm{ETT}} \mathrm{VS} \mathrm{ALI}_{\mathrm{ETT}}{ }^{\mathrm{c}}{ }^{\mathrm{c}}$ indicates $p<0.05 \mathrm{ALI} \mathrm{ETT}_{\mathrm{VS}} \mathrm{ALI} \mathrm{IIV}_{\mathrm{V}}$ 


\begin{tabular}{|l|l|l|l|l|}
\hline & $\mathrm{HL}_{\mathrm{CTRL}}$ & $\mathrm{HL}_{\mathrm{ETT}}$ & $\mathrm{ALI}_{\mathrm{ETT}}$ & $\mathrm{ALI}_{\text {NIV }}$ \\
\hline $\begin{array}{l}\text { Over-distended lung } \\
\text { tissue \% }\end{array}$ & 0 & 0 & 0 & 0 \\
\hline Normally aerated \% & $32 \pm 16$ & $28 \pm 16$ & $78 \pm 26^{\mathrm{a}, \mathrm{b}}$ & $66 \pm 27^{\mathrm{a}, \mathrm{b}}$ \\
\hline Poorly aerated \% & $60 \pm 13$ & $57 \pm 11$ & $16 \pm 15^{\mathrm{a}, \mathrm{b}}$ & $27 \pm 20^{\mathrm{a}, \mathrm{b}}$ \\
\hline Atelectatic \% & $8 \pm 6$ & $15 \pm 15$ & $5 \pm 11$ & $7 \pm 9$ \\
\hline $\mathrm{V}_{\mathrm{L}}(\mathrm{ml})$ & $47 \pm 10.5$ & $38.2 \pm 6.5$ & $77.8 \pm 16.7^{\mathrm{a}, \mathrm{b}}$ & $65.3 \pm 17.5^{\mathrm{a}, \mathrm{b}}$ \\
\hline
\end{tabular}

Table 2: Group mean distribution of lung aeration and lung volume for the different conditions after five minutes of ventilation at $\mathrm{CNANA}_{\mathrm{AL}}$. $\mathrm{HL}_{\mathrm{CTRL}}=$ healthy lung control; $\mathrm{HL}_{\mathrm{ETT}}=$ healthy lung with endo-tracheal tube; $A L I_{E T T}=$ after acute lung injury with endotracheal tube; $A L I_{N I V}=$ after acute lung injury and non-invasive ventilation with nasal prong. $V_{L}=$ lung volume. Values are mean \pm SD.
${ }^{a} \mathrm{p}<0.05$ vs $\mathrm{HL}_{C T R L}$
${ }^{b} \mathrm{p}<0.05$ vs HLETT 